\title{
La cultura de la ciencia: contribuciones para desarrollar competencias de pensamiento científico en un encuentro con la diversidad
}

\author{
The culture of science: contributions to \\ develop scientific thinking skills in a meeting with the diversity
}

Artículo recibido: marzo de 2011

Artículo aprobado: julio de 2011
Silvio Fernando Daza Rosales

Mario Roberto Quintanilla Gatica ${ }^{* *}$

José Rafael Arrieta Vergara ${ }^{* * *}$

"Una revolución supone el abandono de una estructura teórica y su reemplazo por otra incompatible con lo anterior"

Tomas Khum

\section{Resumen}

El presente artículo resalta la importancia y el papel de las ciencias, como actividades humanas complejas, en el campo de las aplicaciones dentro del contexto escolar, teniendo en cuenta los valores del alumnado y los objetivos de la escuela. Por lo tanto, es de suma importancia plantear competencias científicas, definidas como integraciones de conocimientos, habilidades, actitudes, valores y responsabilidades. Para lograr una reflexión más profunda, se realiza una serie de preguntas que deben tenerse en cuenta cuando se construye un currículo de ciencias.

\section{Palabras clave}

Competencia, estándares, ciencia escolar, alfabetismo científico, currículo.

\section{Abstract}

The present article emphasizes on the importance and the role of the sciences, as complex human activities, in the field of applications inside the school context, keeping in mind the values of the pupil and the objectives of the school. Therefore, the importance of outlining scientific competitions, defined as integrations of knowledge, abilities, attitudes, values and responsibilities. In order to achieve a more profound view, a series of questions are made that should be kept in mind when a curriculum of sciences is build.

* Escuela de Agronomía. Instituto Universitario de la Paz-Colombia. Director del grupo de investigación para la renovación de la enseñanza de las ciencias (GRECI). biosidaza 52@hotmail.com

** Profesor de la Facultad de Educación Pontificia Universidad Católica de Chile y director del Laboratorio de Investigación en Didáctica de las Ciencias (GRECIA). mariorqg@gmail.com

*** Escuela de Agronomía. Instituto Universitario de la Paz-Colombia. Director del grupo de investigación de cultivos tropicales (INYUBA) llanadero@hotmail.com 


\section{Keywords}

Competition, standard, school science, scientific literacy, curriculum.

\section{Introducción}

"La ciencia es una de las contribuciones más importanteS de la gran aventura intelectual de las sociedades humanas a lo largo de su historia, lugar donde se concreta la curiosidad y los incansables intentos de representar el mundo en el que construimos y vivimos" (Chamizo, J., 2007).

Son numerosos los autores que consideran la ciencia como parte fundamental de la cultura y la educación, ya que la gran mayoría de los estudiantes sienten curiosidad por el mundo que les rodea y necesitan puntos de referencias para encontrar su propia identidad. Para los docentes, es fundamental preguntarse ¿por qué la necesidad de la cultura de la ciencia, en lo que Fumagalli (2002: 15-35) se plantea ¿Por qué enseñar ciencia en la escuela de primaria? En la necesidad de la cultura de la ciencia en edades iniciales está enmarcado el derecho de los niños a aprender ciencia; aquí se hace un reconocimiento a la Psicología cognitiva y, especialmente, a la psicología genética, que ha aportado valiosa información sobre el modo cómo los niños construyen conocimiento y significado del mundo. Para Bateman, (1999), el niño aprende de ese mundo exterior utilizando todos los órganos sensoriales para ayudarse.

El niño crea constructos mentales para este mundo a medida que el aprendizaje continúa, por consiguiente puede imaginar cosas y hechos. Él interactúa con ese ambiente exterior creando estructuras mentales a lo largo de una etapa en la que se aprende a mirar las cosas, a contarlas, a organizarlas por categorías y nociones, a recordar y disfrutar recordando, a hacer cálculos mentales, a imaginar que pasaría "si", y, aún así, a diferenciar las situaciones reales de las inventadas o imaginarias que les sirven para resolver problemas. Sin embargo, hay un amplio conjunto de investigaciones (Harlen, W., 1999) que manifiestan que, si no se interviene para introducir un enfoque científico en su exploración del mundo, es fácil que las ideas que elaboren los niños sean acientíficas y dificulten el aprendizaje de las ciencias en secundaria y en la universidad.

La escuela volvió a considerarse como la institución social encargada de distribuir en la población un conjunto de contenidos culturales que no son capaces de transmitir y generar los grupos primarios (tales como la familia), ni los medios de comunicación social ni el desarrollo espontaneo del niño en la vida colectiva (Pérez, A.,1992).

El niño no es una abstracción, él responde a una organización social real, a un tipo de formación cultural histórica y, por lo mismo, hay que pensarlo de manera concreta como un constructo orgánico, sensible, afectivo, imaginativo, creativo y estimulado siempre por el asombro que le producen las cosas del mundo externo, las cuales lo predisponen al interés por conocer la ciencia desde una edad temprana.

Los niños tienen ideas y las ponen en práctica, desarrollan teorías que constantemente convierten en acción, utilizan distintos lenguajes para expresarlas, las examinan y reexaminan, proceso en el que los adultos somos participantes activos. La idea de «niño y niña competente» expresada por Zabalza (1996) es interesante para entender lo que los niños de educación infantil traen a la escuela y también las experiencias y destrezas con las que salen. Este es un buen argumento para entender las potencialidades de la infancia.

Las prácticas reiteradas en el conocimiento, estimuladas por procesos científicos graduales, hacen de el niño un ser en capacidad de desarrollar ejercicios adiestrados que van a traducirse en habilidades. Con ellas, la destreza manual-cerebral que lo ha de posibilitar en un mundo de mayores exigencias cognitivas de tipo científico es también desarrollada.

La adopción de esta perspectiva nos lleva a un modelo poco estable comparado con la visión del conocimiento como cultura universal. Sin embargo, ofrece la posibilidad de tomar decisiones sobre el aprendizaje en un contexto, la escuela infantil, en 
la que los eventos ocurren sin prisa y sin sujeción a las presiones de la enseñanza obligatoria.

La escuela infantil se convierte así en un espacio idóneo para que niños y adultos exploren nuevas posibilidades de conocimiento, trabajen de forma creativa en la materialización de sus descubrimientos y se les posibilite la expresión de las ideas mediante la utilización de lenguajes diversos.

El conocimiento y la cultura se (re)construyen en relación con las otras personas. Es lo que Malaguzzi (2001) denomina pedagogía de las relaciones haciéndolo coincidir con la pedagogía del aprendiza$j e$. Lo relacional explica perfectamente la existencia de individuos e identidades particulares. Individuo y grupo configuran una escuela en la que confluye lo objetivo y lo simbólico, las representaciones o las relaciones entre niños y adultos.

Lo individual se construye a partir de las conexiones con distintos grupos. Pero este pensamiento no es solo un instrumento didáctico para organizar el trabajo en la escuela. Se trata de comprender que el diálogo, las relaciones y los encuentros deben de formar parte de la cultura cotidiana en la escuela infantil.

Ese conjunto de contenidos culturales que constituye el cuerpo de conocimientos escolar es público (Stenhouse, W., 1985), en el sentido de que ha sido elaborado y sistematizado socialmente. Asignarle a la escuela el papel social de distribuir dichos contenidos supone reconocer que el lugar social de pertenencia, aunque fuente de producción cultural, no garantiza el acceso al conocimiento de la cultura elaborada por el cuerpo social. La escuela es por ahora el ámbito que podría posibilitar de manera adecuada este acceso y es la responsable de distribuir socialmente los contenidos que la cultura elabora a partir del conocimiento científico en las ciencias naturales, las cuales forman parte del capital cultural básico de la población.

En la Declaración de la Conferencia Mundial sobre Ciencia para el siglo XXI, auspiciada por la UNESCO y el Consejo Internacional para la Ciencia, se expresa: "Para que un país tenga la capa- cidad de abastecer las necesidades básicas de su población, la educación en ciencia y tecnología es una necesidad estratégica". Como parte de esa educación, los estudiantes deben aprender a resolver problemas específicos y a responder a las necesidades de la sociedad, utilizando el conocimiento y las habilidades científicas y tecnológicas.

El crecimiento vertiginoso del conocimiento científico y la adopción masiva de innovaciones tecnológicas, ocurridas durante el siglo XX y en particular en la primera década del siglo XXI, han tenido y tienen una fuerte influencia sobre nuestros estilos de vida y nuestro propio bienestar. Estos avances han promovido cambios en la manera de vernos como personas, de pensar, de comunicarnos y de trabajar. Sobre todo, han generado un profundo cambio en nuestra ubicación e interacción con la naturaleza.

Los nuevos avances en la ciencia tienen su correlato en el desarrollo de nuevas tecnologías, cubriendo áreas que abarcan desde la salud (desarrollo de nuevos fármacos, introducción de técnicas no invasivas de análisis, avances en microcirugías, etc.), biotecnología (nuevas formas de producción no contaminantes), entre otros. Y estas a su vez promueven nuevas disciplinas no concebidas hace un cuarto de siglo.

Para poder progresar en este nuevo escenario, se necesita formar personas creativas, capaces de desarrollar nuevas ideas, de identificar y resolver problemas; personas comprometidas con el destino común de sus semejantes, interesados en construir, en compartir, en producir y en ser capaces de adaptarse rápidamente a dichos cambios.

En virtud de la velocidad con que se producen los avances, la adaptación a los cambios es uno de los aspectos centrales sobre los cuales debería articularse el proceso de formación desde los primeros estadios de la educación.

Por consiguiente esa formación estaría enmarcada en educar para la competencia... Según expuso Delors en una intervención memorable en referencia a la orientación de la enseñanza en la Unión Europea, "la educación ha de ser desarrollo de 
competencias que corresponden a cuatro dimensiones humanas: la del ser, la del hacer, la del conocer y la de convivir".

A un alumno "competente", "los conocimientos" le ayudan a "hacer", en sintonía con su propio "ser" (su creatividad), a la vez que convive con los otros (porque sabe trabajar en equipo). Va emergiendo un nuevo enfoque de la enseñanza y una nueva manera de evaluar a los alumnos, por competencias (Harlen, 2002; Bruner, 1999).

Este cambio propuesto requiere un punto de partida para la enseñanza de las ciencias muy diferente del actual, lo que interesa es que "lo que se sabe" sea, a la vez, lo que se puede hacer, lo que se evalúa de manera personal y lo que se realiza con los otros. A partir de este punto de partida, las ciencias dejan de ser neutras; aparecen de manera muy natural numerosos sistemas de valores que intervienen $y$, con ellos, se hace necesario el espacio de libertad que permite escoger a cual no se puede renunciar.

\section{¿Por qué hay que enseñar las ciencias como cultura en la cultura de la diversidad?}

La cultura es una visión de mundo ecúmene que no se agota con el arte y la literatura, es un concepto más complejo y totalizador del quehacer humano que construye mundos más allá de la estética y de cuya dinámica siempre posibilita escenarios y discursos que den validez y justificación a sus diversas ramificaciones del saber y del conocimiento científico. Desde el más remoto pasado la cultura es concebida como siembra en tierra fértil, y por metonimia pasa a ser sembradura del pensamiento. Cultivar la tierra con la siembra de la semilla articula un enfoque semiológico donde la palabra hace de agrimensura (Fara, P 2009) ${ }^{1}$.

Con Cicerón se habló de la "cultura animi", también conocida como cultura del espíritu en la an-

1 Fara (2009) plantea que la palabra geometría significa medir la tierra y que los matemáticos griegos contribuyeron a convertir los problemas de agrimensura práctica en diagramas abstractos. Utilizando al principio las técnicas ya conocidas por los niños babilonios, desarrollaron poco a poco un conocimiento matemático teórico fascinante por sí mismo, aparte de su valor práctico. tigua Roma pero concebida como cultivo de la tierra. Cultivar el espíritu es un tropo metonímico que viene a significar el fruto del pensamiento. Aquí pues, está implicado ya una relación del mundo físico devenido en mundo espiritual revestido desde la cultura. En otras palabras, puede colegirse de manera reposada, reflexiva, que el mundo de la cultura siempre ha estado presente en las prácticas discursivas de las ciencias, y con mayor presencia en la esfera de las ciencias naturales.

Desde luego, el término cultura está vinculado orgánicamente en la historia de las ciencias naturales, por lo tanto, participa trascendiendo la primigenia imagen de arte (imitación de la naturaleza), para ser pensada y difundida con su relación orgánica con la tierra. Entonces, cultura, arte y vida cotidiana son eslabones de una historia científica que encuentra en las ciencias naturales un espacio abonado ya no como aventura del pensamiento, sino como una ciencia que encuentra en la vida misma su razón de ser. Estética en sí misma, también es vida cotidiana desde la intimidad del hogar donde crece el niño y es educado.

Esta vida cotidiana transita hacia la escuela para ampliar su horizonte de posibilidades cognitivas con base en las ciencias, de manera específica, en el campo de las ciencias naturales. Se puede decir, en términos de axiología que el máximo de los valores es la vida, lo que permite inferir que, con la vida se puede hacer estética de lo cotidiano y discurso científico. En pocas palabras Verso y Universo están mediados por la palabra. La palabra como semilla y la tierra como surco, para que en virtud de las leyes orgánicas del suelo, germine el fruto; de esa manera, se puede hablar de una estética de la vida cotidiana y de la ciencia biológica.

Para la construcción de la cultura de la ciencia, como contribución a la promoción de competencias de pensamiento científico, estarían enmarcados los valores principales de la ciencia y la necesidad de contribuir a la comprensión del mundo que rodea a los niños y jóvenes (considerando la comprensión como estructura mental en desarrollo que cambia a medida que se amplía la experiencia infantil). La capacidad de desarrollar formas, de 
descubrir cosas, comprobar ideas y utilizar pruebas; el modo en que los niños interactúan con las cosas que les rodean y que apoyan su aprendizaje, no solo en ciencias, sino también en otras áreas.

Comprometernos con la cultura de las ciencias es invitar a los niños a mirar el mundo con otros ojos, transformando aspectos de sus cotidianidades en contenidos para ser enseñados, brindando oportunidades para explorar y pensar el mundo desde otra perspectiva, con los anteojos de las ciencias.

Ampliar sus horizontes culturales, propiciando el conocimiento de diversas realidades cercanas y lejanas en el tiempo y en el espacio, que al ser provistas por la familia, seguirán siendo desconocidas para ellos de no mediar la intervención escolar.

Desarrollar habilidades cognitivas lingüísticas, promoviendo situaciones que incentiven la oralidad, en las que deban preguntar, contar, opinar, intercambiar información, explicar o comparar y usar el vocabulario científico. Recordemos que el uso del lenguaje también es un medio que les permite ir apropiándose de los nuevos conocimientos.

La oralidad no es tan pasajera como algunas personas suponen. La expresión oral es una manera creativa de decir y nominar las cosas desde un espacio y pertenencia cultural. Las culturas ancestrales privilegiaron la palabra como forma de memoria en las voces y andaduras de los mayores. Con ellos se siembra una tradición de ancestralidad en los saberes inmateriales de cultura que preservan y divulgan a los niños/niñas como legítimos herederos de los conocimientos y cosmovisiones del pasado.

La ciencia como cultura es un estilo de pensamiento y de acción que nos permite no sólo mirar nuestro mundo, sino que tratar de comprenderlo. El principal objetivo de la investigación científica es incrementar nuestro conocimiento (objetivo intrínseco o cognitivo) y aumentar nuestro bienestar y nuestro poder (objetivo extrínseco o utilitario). La ciencia tiende a construir reproducciones conceptuales de las estructuras de los hechos. Son los conceptos los que están en la base de la ciencia.
El comprender la función y constitución de la membrana plasmática, el conocer los componentes y estructura de la molécula de agua o conocer la intensidad de diferentes sonidos sólo son significativos, y por lo mismo relevantes, en la medida en que nos permiten comprender el funcionamiento y cuidado de nuestro cuerpo; o aprender a valorar el agua como un recurso y saber cómo utilizarlo; o distinguir aquellos sonidos que por su intensidad pueden ser dañinos para el oído humano. En fin, este conocimiento es relevante, es significativo en el grado que permite comprender nuestro entorno, $y$ nos permite responder y generar nuevas preguntas.

Esta comprensión de nuestro entorno, pasa por relacionar lógicamente conceptos en el marco de teorías. Son las aplicaciones de estas teorías las que nos permiten explicar y predecir fenómenos naturales.

Las ciencias constituyen una manera de pensar y de actuar con el objetivo de interpretar determinados fenómenos e intervenir en ellos mediante un conjunto de conocimientos teóricos y prácticos, estructurados. El Modelo Cognitivo de Ciencia (MCC) nos muestra que el proceso mediante el cual se construyen estos conocimientos no es radicalmente distinto del que se da en otras elaboraciones humanas con las cuales se da significado a los acontecimientos que se quieren controlar (Giere, 1988; 1992). El conocimiento científico tiene la peculiaridad de poder estar al alcance de todos aquellos que quieren saber cómo funciona el mundo y cómo intervenir en él.

La ciencia es una parte importante de nuestra cultura porque ofrece un conjunto de historias explicativas y sustantivas que nos cuentan cosas nuevas, importantes e interesantes sobre nosotros mismos y el mundo en que vivimos. Sin duda, éstas han demostrado ser perfectamente fiables y útiles. Por ejemplo, ahora nos beneficiamos ampliamente de conocimientos como los siguientes: muchas enfermedades las trasmiten los microorganismos; el ojo solo no puede ver; los rasgos hereditarios son transmitidos por un código químico; todas las especies han evolucionado a partir de organismos más simples; todas las sustancias están compuestas de partículas diminutas, unidas por fuerzas de 
naturaleza eléctrica; muchas sustancias distintas que vemos están formadas por diferentes redisposiciones de las mismas partículas.

Si las ciencias son el resultado de una actividad humana compleja, su enseñanza no puede ser menos: debe concebirse también como actividad y para ello debe tener la meta, el método y el campo de aplicaciones adecuados al contexto escolar, conectados con los valores del alumnado y con el objetivo de la escuela, promover la construcción de conocimientos y hacerlos evolucionar (Sanmartí e Izquierdo, 1997).

Una razón fundamental para enseñar la ciencia a los jóvenes es transmitirle algunos de estos conocimientos sobre el mundo material, sencillamente porque es a la vez interesante, apasionante e importante. No es el tipo de conocimiento que se adquiere sencillamente por experiencia, sino que se debe ofrecer mediante una enseñanza cuidadosamente programada porque las escuelas siguen siendo el principal agente de reproducción cultural. Carecer de conocimientos científicos significa, en un sentido muy real, ser un marginado, tan extraño a nuestra cultura como alguien nunca haya oído hablar de Gabo.

Enseñar ciencias ha sido desde siempre, y seguirá siendo, un gran desafío; el problema es el manejo ineficiente de los conceptos y de sus significados, generando confusiones tales como: peso con masa, volumen con masa. Existen muchos ejemplos, producidos no solo por una escasa formación del área, sino también por una metodología y un uso del método científico desvirtuado.

Frente a lo anterior, la Teoría de los modelos de Giere $(1988,1992)$, puede encontrar un camino apropiado y educativo para la transposición del saber erudito en la clase de ciencias, que parece ser muy interesante y prometedor para enfrentar la evaluación como un proceso de desarrollo del sujeto que aprende. Giere permite establecer prudentes conexiones entre los modelos teóricos (el mundo de las ideas), y el sistema real (trabajo experimental, manipulación de lo real) del estudiantado, los profesores y los científicos. Buscando establecer simi- litudes a través de las hipótesis teóricas y las entidades lingüísticas que los relacionan (Giere, 1999; Quintanilla, 2006).

De esta forma, se piensa, se hace y se expresa la ciencia como cultura que conecta permanentemente estos tres elementos, sin separar la teoría de lo empírico, ya que asumimos la elaboración de modelos teóricos de los fenómenos con que estamos trabajando (modelo fenomenológico); de los instrumentos que estamos usando (modelo instrumental) y de nuestra intervención sobre el mundo real (modelo cotidiano).

Uno de los elementos interesantes que postula este modelo de ciencia, es el racionalismo hipotético, que significa que si yo tengo una meta, voy hacia ella planteando cómo hacerlo "en el camino". En definitiva, las comunidades científicas y las personas saben valorar si se acercan bien o mal a la meta, sobre la base de una construcción en común de un hecho paradigmático y de la interpretación del mismo sobre la base de diferentes miradas del mismo fenómeno (diversidad de explicaciones científicas en el alumnado). En este sentido, el estudio de cómo funciona una comunidad, cuáles son los "criterios de racionalidad", etc., nos acercan a una "Racionalidad Moderada" para comprender la ciencia que enseñamos y la finalidad que impregnamos a la evaluación de competencias de pensamiento científico en esta variante epistemológica.

Por tanto, creemos que una de las finalidades principales de la educación científica de hoy en día es el lograr que niños y niñas, adolescentes y jóvenes sean capaces de poner en marcha, de forma autónoma y crítica, estos procesos cognitivo lingüísticos (Sanmartí, 2003) para dar coherencia a su pensamiento científico (teórico), su discurso y su acción sobre el mundo natural; con el fin de poder darle sentido, intervenir activamente en él, tomar decisiones fundamentadas y establecer juicios de valor robustos que contribuyan a la cultura ciudadana y a la promoción de la paz. En este sentido, es ineludible el abordaje del sistema de competencias de pensamiento científico y cómo ellas aparecen en el proceso formativo; es decir, la formación del pensamiento como tarea pedagógica y didáctica, que no 
puede realizarse plenamente si no se cuenta con el sistema de situaciones y dispositivos de evaluación que hagan factible tanto la identificación como el correspondiente proceso formativo y formador.

Es indiscutible que tal tarea solo puede llevarse a cabo satisfactoriamente apoyados en una concepción y una acción didáctica formativa que incorpore al aprendizaje de las ciencias naturales, en todos los niveles de escolaridad (desde la etapa inicial hasta el universitario), una manera diferente de proceder a como ha venido haciéndose. Las situaciones evaluativas deben ser reales y profundamente educativas, incluso emancipadoras; es decir, que formen personas con las capacidades de saber, hacer, ser y vivir en comunidad. En esta línea, creemos necesaria la recuperación de un "modelo de ciencia ( $y$ evaluación del pensamiento científico) como actividades profundamente humanas": hechas por humanos y para humanos (Izquierdo, 1996, 2000).

Así, el conocimiento científico adquirido por el estudiantado debería ser persistente y significativo. De acuerdo a teorías modernas y tendencias internacionales sobre desarrollo de competencias de pensamiento científico y modelos de evaluación, esto se lograría a través de un proceso de construcción activo y protagónico al interior de cada sujeto y en el contexto sociocultural donde sus competencias adquieren significado $y$, por tanto, son evaluadas en función de las tareas y los contextos culturales en que se inscribe. En este contexto, las concepciones previas resultarían ser de carácter esencial para la comprensión de las teorías y modelos explicativos de la ciencia, de su método y su naturaleza.

Del mismo modo, es necesario que las prácticas de evaluación de los docentes de ciencias naturales posibiliten a los estudiantes evidenciar cómo van logrando acceder al mundo de las ciencias, no solamente por la vía de los productos que se presentan, sino que también por la vía de los procesos que se dan para lograr adquirir un determinado conocimiento. Así, las cualidades personales y grupales consustanciales les permitiría generar más instan- cias de autorregulación con respecto a la calidad de sus aprendizajes, identificando logros, criterios, obstáculos, etc.; potenciando así competencias y habilidades científicas que los preparan para la vida y para el éxito profesional en lo que emprendan.

Todo lo anterior inscribe nuestro proyecto de formación continua de profesores de ciencias en un contexto de aprendizaje superior, que propicia una conducta personal y socialmente responsable en la comprensión de la complejidad de la ciencia en el mundo que marca el devenir actual de la sociedad y la cultura científica ciudadana.

Uno de los factores que lleva al estudiante a ver con dificultades la ciencia se relaciona con procedimientos pedagógicos tradicionales, en donde se enseña como un dogma con leyes, principios y fundamentos que son prácticamente incuestionables. Esta manera de ver y enseñar las ciencias ha predominado en nuestras aulas durante años; pero se hace incompatible con una sociedad en que el conocimiento científico y sus aplicaciones, muchas en el campo de la tecnología, están estrechamente vinculadas con sus requerimientos sociales y viceversa; El propio conocimiento se genera, destruye y reconstruye a ritmos nunca antes vistos, por lo que se requiere personas capaces de adaptarse a estos cambios y entender, no memorizar, los conceptos asociados a las ciencias y al estudio de éstas.

La noción de competencia surge como una necesidad de respuesta a nuestra sociedad, de la capacidad individual de tener habilidades y enfrentar el contexto globalizado.

Se espera que los alumnos se cuestionen, no que memoricen y entreguen datos; que desde temprana edad se destaquen por sobre las demás comunidades educativas por su carácter crítico, pensante, tecnológico, competente y habilidoso en destrezas teóricas y prácticas; que puedan predecir basados en lo que construyen como formas de conocimiento potenciadas desde el área de la experimentación bien orientada, no buscando comprobar fenómenos que ya sabemos que son (ese no es el norte de una experimentación). 
La ciencia nos permite mirar nuestro mundo y tratar de comprenderlo, ahí está su objetivo último. Y las fórmulas y largas listas de conceptos no son otra cosa que herramientas que nos permiten abordar y manejar los conceptos. Son los modelos teóricos los que están en la base de la ciencia.

Es de considerar que al aplicar la metodología de la investigación científica de manera correcta, es decir, con el carácter de la experimentación no en blanco ni por la mera diversión de ver fenómenos entretenidos, sino con la intención por parte del docente de plantear problemas en torno al eje temático (acorde a la malla curricular y los mapas de progreso). De tal forma que el alumno aplique sus bases conceptuales, en torno a la experimentación, estructurado en la lógica del método científico para plantear una comprobación a sus hipótesis. El estudiante busca generar un nuevo conocimiento o una nueva pregunta, conocer cuál es su nivel de abstracción para potenciar dicho proceso (generación de conocimiento científico), de cómo se hace y se aprende la ciencia. Se hace necesario entonces diseñar escalas de abstracción, medirlas y potenciarlas, todo fundamentado desde la lógica de la ciencia.

Desde este punto de vista metodológico, los conceptos son instrumentos utilizados para distinguir entidades y agruparlas; ellos nos permiten realizar el análisis y la síntesis de manera conceptual y empírica. En particular, los conceptos individuales nos sirven para distinguir entre individuos, y los conceptos de clases para establecer clasificaciones. Algunos conceptos relacionales posibilitan la comparación y la ordenación, y los conceptos cuantitativos son el núcleo conceptual de la medición.

Por consiguiente, la ciencia escolar ha de «tener valor» para los alumnos, porque sólo así harán de ella una actividad significativa, sólo así podrán «entrar en el juego» y aprenderla. Vivimos en una sociedad humanista donde la emoción, más que la razón, es la que configura los pensamientos y actos de las personas, donde se aprecia mucho la individualidad y la expresión y la creatividad personal, donde las palabras y las imágenes son más importantes y superiores que las ideas.
Fijar el valor que la ciencia tiene tanto para la persona como para nuestra cultura implica dos cosas: recuperar la dimensión social, histórica y cultural, en resumen, el aspecto humano; y en segundo lugar, reconocer que la ciencia no goza de privilegios epistemológicos, que no es algo bueno e incuestionable, que no pueden censurar ni cuestionar los inexpertos que no sea miembros de derecho del club de las ciencias. Solo si se comprende la naturaleza de la sociedad en la que se asienta y la naturaleza de su relación cambiante con el público de esa sociedad, se puede iniciar una revaloración de la ciencia.

\section{Un caso particular para una ciencia de todas y todos...}

En las últimas décadas el currículo de ciencias naturales ha sufrido cambios importantes, motivados por los siguientes factores:

- Los nuevos objetivos de la educación básica y media, definidos en las sucesivas reformas del sistema educativo, que enfatizan los objetivos de alfabetización científica de los estudiantes y la adquisición de competencias.

- Los resultados de la investigación en didáctica de las ciencias.

- Las nuevas experiencias de innovación curricular en ciencias naturales.

- El desarrollo de las propias disciplinas.

En los primeros años del siglo XXI muchos países han proseguido procesos de reformas de sus sistemas educativos y de revisión de curriculum de ciencia, poniendo el énfasis en la adquisición de competencias y en la formación científica del estudiante, con el objetivo de alcanzar niveles satisfactorios de alfabetización científica para todo el alumnado (Osborne, et al., 2003).

Frente a esto, se han elaborado proyectos internacionales como el PISA y TIMMS que pretenden evaluar el nivel de formación científica de los estudiantes, definido como la formación científica (termino equivalente al de literancia científica o alfabetización científica), es decir, la capacidad de utilizar el conocimiento científico para identi- 
ficar preguntas y obtener conclusiones a partir de pruebas, con la finalidad de comprender y ayudar a tomar decisiones sobre el mundo natural y los cambios que la actividad humana produce en él. Así, se considera que los estudiantes han de estar preparados para la comprensión de la naturaleza de la ciencias, de sus procedimientos, de sus puntos fuertes y sus limitaciones, así como el tipo de preguntas a las cuales puede responder.

También se considera importante que los estudiantes sean capaces de argumentar y comunicar eficazmente sus conocimientos a audiencias concretas y que puedan tener opiniones fundamentadas, participar en los temas que se discuten en la sociedad... Sin embargo, partamos de que existe, en muchas de nuestras comunidades, "la errónea convicción de que la "mollera" está hundida porque el niño tiene "mal de ojo", lleva a prácticas peligrosas como colgar al menor de los pies y golpearlo en las plantas de los pies para "corregir" la fontanela. En otros muchos casos el sobador succiona con fuerza la fontanela para darle la forma original".

En una sociedad caracterizada por una cultura tan rica en leyendas, mitos, costumbres, tradiciones y cuya población cuenta con un considerable índice de analfabetismo y baja escolaridad promedio, es comprensible que los casos antes mencionados lejos de disminuirse se mantengan constantes y en ciertos momentos incluso con tendencia al alza.

Mientras el mundo ha caído en cuenta de que se necesita urgentemente hablar un segundo idioma (inglés de preferencia) y aprender el uso y aplicación de la computadora en la vida estudiantil, profesional, laboral y cotidiana, parece haberse olvidado de la importancia del alfabetismo científico, de una ciencia de todas y para todas.

Frente a lo anterior, Mercé Piqueras citando a Carl Sagan (1934-1996) plantea que el analfabetismo científico fue definido por el astrofísico y divulgador científico como "la incapacidad de comprender los mecanismos más sencillos de la ciencia, tanto los conceptos científicos como sus objetivos y los procedimientos de la ciencia”.
Lo paradójico es que aún cuando la ciencia y el conocimiento científico no son considerados "urgentes" para la vida, vivimos en un mundo que no puede prescindir de la ciencia que promueve la tecnología, nosotros mismos en la vida cotidiana nos encontramos rodeados de fenómenos y procesos que tienen explicación científica.

Tal y como dice Pilar Durá Gilabert “... Desde el móvil a la televisión, desde los antibióticos a la anestesia. Nuestro bienestar es totalmente dependiente de los productos de la ciencia y la tecnología, cada vez en mayor medida. Paradójicamente, cada vez sabemos menos y menos sobre ciencia y tecnología".

\section{Las competencia en la cultura de pensamiento científico...}

Paralelamente, en el mundo globalizado en el que habitamos, las sociedades han comprendido la necesidad de fortalecer en su población habilidades, conocimientos, valores y actitudes que les permitan un mejor posicionamiento y participación social, enfrentando de manera competente las oportunidades y retos que se presenten. Esto incluye estrategias para fortalecer el alfabetismo científico, como parte de esas estrategias, entre los cambios que proyecta, está el aprendizaje por competencias.

La noción de competencia científica según Quintanilla (2006), nos remite a alguien que es capaz, que sabe, que tiene la habilidad reconocida para afrontar una situación, que posee un cierto grado de dominio y recursos. Es alguien que ha desarrollado las acciones de captar, pensar, explorar, atender, percibir, formular, manipular e introducir cambios que permiten realizar una interacción competente en un medio dado o especifico.

Las competencias representan una combinación dinámica de atributos en relación a conocimientos, habilidades, actitudes, valores y responsabilidades que describen los resultados de aprendizaje dentro de un programa educativo mucho más amplio y enriquecedor, en que los alumnos son capaces de demostrar de manera no reproductiva que han aprendido ciencia. 
Con el desarrollo de estas competencias en el estudiantado, no se pretende que las futuras generaciones sean científicos, pero sí que la población mejore su calidad de vida y la capacidad de relacionarse con el medio que le rodea. Por ejemplo, entender lo que la ciencia explica acerca del funcionamiento del cuerpo humano, o por qué nuestro organismo manifiesta cierto tipo de respuestas. Al estudiar fenómenos físicos y químicos tales como los cambios que sufre la materia, podemos reconocer su presencia en la actividad diaria, en la cocina de nuestros hogares. Al comprender los procesos que permiten avances científicos y tecnológicos podamos aplicarlos a nuestra vida cotidiana, mejorando la criticidad de nuestro pensamiento y la responsabilidad en las decisiones que tomamos.

Lo que se pretende generar en los estudiantes al desarrollar las competencias, es fomentar su formación como pensadores autodirigidos, autodisciplinados y en automonitores. Para esto, es necesario plantear preguntas y problemas esenciales (formulándolos de manera clara y precisa); es preciso recopilar y evaluar información relevante (usando ideas abstractas para interpretarlas de manera efectiva y justa). Llegar a conclusiones y soluciones bien razonadas (comparándolas contra criterios y estándares relevantes), pensar de manera abierta dentro de sistemas de pensamiento alternativo (reconociendo y evaluando, conforme sea necesario, sus suposiciones, implicaciones y consecuencias prácticas), para poder comunicarse de manera efectiva con los demás en la búsqueda de soluciones para problemas complejos.

\section{La indagación como alternativa metodológica de las ciencias en una cultura de la diversidad}

La indagación se puede definir como un "Conjunto de comportamientos involucrados en la búsqueda de explicaciones razonables, de situaciones que despiertan nuestra curiosidad en ciencias. Una estrategia para enseñar y aprender ciencias".

Los docentes del área de ciencias naturales deben tener en cuenta que para proponer la estrategia, la indagación y para promover las CPC son necesarios los siguientes aspectos:
- Usar la complejidad de los problemas de la vida real.

- Usar el error como fuente de aprendizaje.

- Crear ambigüedad y conflicto cognitivo.

- Promover la reflexión y metacognición.

- Promover la discusión entre los alumnos, orientándola a través de preguntas abiertas.

En toda actividad indagatoria se parte de una situación-problema, una pregunta con respecto a un fenómeno concreto que sea interesante de ser analizado e investigado por el estudiante.

Una vez que se formula la pregunta, el estudiante elabora sus propias explicaciones para responder a esta pregunta, de manera que sea posible dar una primera respuesta desde sus conocimientos e intuiciones. Esta primera respuesta (hipótesis), para ser verificada, necesita ser puesta a prueba.

Para poder confirmar o desmentir su hipótesis, el estudiante debe realizar una experiencia concreta que le permita saber si su hipótesis es correcta o no. Una vez finalizada, el estudiante analiza la experiencia realizada, compara sus resultados con su respuesta original y, basada en los datos obtenidos, corrige, reelabora y amplía su respuesta. Esta respuesta, basada en una experiencia concreta, le permite resolver nuevos problemas y plantearse nuevas interrogantes relacionadas con la experiencia realizada.

\section{Aspectos a tener en cuenta para la pro- moción de Competencia de Pensamiento Científico (CPC)}

Hablar de competencias de pensamiento cientifico (el tema que da título a este trabajo) sólo tiene sentido desde esta perspectiva: la de una ciencia que se vive, que se hace, que evoluciona al ritmo de nuevas finalidades humanas que no decae en la capacidad humana de formular interrogantes. ¿Qué entendemos por competencias? Existe ahora una intensa discusión sobre el tema, brevemente se puede decir que una competencia apela a saber hacer, ser y vivir con otros en situaciones la vida en las cuales se ha de decidir cómo actuar. 
Si la competencia tiene que ver con la ciencia, la situación es tal que moviliza conocimientos que no pueden ser «de libro», sino que corresponden a una actividad científica. Así, no se puede aislar el saber científico de la vida, de sus aplicaciones, de sus implicaciones, de su significado en relación con otras materias.

Llegamos así al punto crucial: la ciencia no empieza en los hechos, sino en las preguntas; y las preguntas dependen del marco teórico desde el cual se formulan.

Así, los hechos no son independientes de los observadores y de sus maneras de ver el mundo. La sociedad en que viven día a día la comunidad científica, los docentes y el alumnado (los dos últimos en un proyecto de ciencia escolar) determina o limita el tipo de preguntas que se hacen o que pueden responder ellos mismos; además de influir en sus conclusiones, debido a la presencia o ausencia de programas educativos o de investigación científica, de reconocimientos o castigos a la misma actividad y de tolerancia o imposición de áreas de investigación.

En pocas palabras, las preguntas que la comunidad científica, los docentes y el alumnado se hacen, corresponden a las que tradicionalmente las sociedades o las escuelas aceptan; pero, en la medida en que la escuela actual se abra al futuro y eduque el sentido crítico y la capacidad de decidir razonadamente, pueden emerger nuevas preguntas. Estas nuevas preguntas serán apropiadas para desarrollar competencias y para evaluarlas, pero también harán posible un futuro más creativo, en el cual la ciencia continúe aportando conocimientos sobre el mundo. Como ya se dijo antes, las preguntas concretan los problemas. Por ello, aprender a preguntar es una competencia y aprender a evaluarla es una necesidad.

Por consiguiente, favorecer la promoción y desarrollo de competencias de pensamiento científico para una cultura de la diversidad es fundamental; como afirma Gil Daniel (2005), es necesario que los docentes de ciencias naturales se planteen los siguientes interrogantes:
1. ¿Se presentan situaciones problemáticas abiertas (con objeto de que los alumnos puedan tomar decisiones para precisarlas) de un nivel de dificultad adecuado (correspondiente a su zona de desarrollo próximo)?

2. ¿Se plantea una reflexión sobre el posible interés de las situaciones propuestas que dé sentido a su estudio (considerando su relación con el programa general de trabajo adoptado, las posibles implicaciones CTSA...)?

3. ¿Se presta atención, en general, a potenciar las actitudes positivas y a que el trabajo se realice en un clima próximo a lo que es una investigación colectiva (situación en la que las opiniones, intereses, etc., de cada individuo cuentan) y no en un clima de sometimiento a tareas impuestas por un profesor/"capataz"?

4. ¿Se procura evitar toda discriminación (por razones étnicas, sociales...) y, en particular, el uso de un lenguaje sexista, transmisor de expectativas negativas hacia las mujeres?

5. ¿Se plantea un análisis cualitativo, significativo, que ayude a comprender y a acotar las situaciones planteadas (a la luz de los conocimientos disponibles, del interés del problema, etc.) y a formular preguntas operativas sobre lo que se busca?

6. ¿Se muestra, por otra parte, el papel esencial de las matemáticas como instrumento de investigación, que interviene desde la formulación misma de problemas, al análisis de los resultados, sin caer en operativismo ciegos?

7. ¿Se plantea la emisión de hipótesis, fundamentadas en los conocimientos disponibles, susceptibles de orientar el tratamiento de las situaciones y de hacer explícitas, funcionalmente, las preconcepciones?

8. ¿Se presta atención a las preconcepciones (que, insistimos, deben ser contempladas como hipótesis) y a la actualización de los conocimientos que constituyen prerrequisitos para el estudio emprendido?

9. ¿Se plantea la elaboración de estrategias (en plural), incluyendo, en su caso, diseños experimentales? 
10. ¿Se presta atención a la actividad práctica en sí misma (montajes, medidas...), dando a la dimensión tecnológica el papel que le corresponde en este proceso?

11. ¿Se potencia la incorporación de la tecnología actual a los diseños experimentales (ordenadores, electrónica, automación...), con objeto de favorecer una visión más correcta de la actividad científico-técnica contemporánea?

12. ¿Se plantea el análisis detallado de los resultados (su interpretación física, fiabilidad, etc.) a la luz del cuerpo de conocimientos disponible, de las hipótesis manejadas y/o de los resultados de otros equipos?

13. ¿Se plantea una reflexión sobre los posibles conflictos entre algunos resultados y las concepciones iníciales (conflictos cognitivos), favoreciendo la "autorregulación" del trabajo de los alumnos?

14. ¿Se promueve que los estudiantes cotejen su evolución conceptual y metodológica con la experimentada históricamente por la comunidad científica?

15. ¿Se plantea la consideración de posibles perspectivas (replanteamiento del estudio a otro nivel de complejidad, problemas derivados...)?

16. ¿Se consideran, en particular, las implicaciones CTSA del estudio realizado (posibles aplicaciones, repercusiones negativas, toma de decisiones...)?

17. ¿Se pide la elaboración de "productos" (prototipos, colecciones de objetos, carteles,...) poniendo énfasis en la estrecha relación ciencia-tecnología?

18. ¿Se pide un esfuerzo de integración que considere la contribución del estudio realizado a la construcción de un cuerpo coherente de conocimientos, las posibles implicaciones en otros campos de conocimientos, etc.?

19. ¿Se pide algún trabajo de construcción de síntesis, mapas conceptuales, etc., que ponga en relación conocimientos diversos?
20. ¿Se presta atención a la comunicación como aspecto esencial de la actividad científica?

21. ¿Se plantea la elaboración de memorias científicas del trabajo realizado?

22. ¿Se pide la lectura y comentario crítico de textos científicos?

23. ¿Se presta atención a la verbalización, solicitando comentarios significativos que eviten el "operativismo mudo"?

24. ¿Se potencia la dimensión colectiva del trabajo científico organizando equipos de trabajo y facilitando la interacción entre los equipos y la comunidad científica (representada en la clase por el resto de los equipos, el cuerpo de conocimientos ya construido, los textos, el profesor como experto...)?

25. ¿Se hace ver, en particular, que los resultados de una sola persona o de un solo equipo no pueden bastar para verificar o falsar una hipótesis?

26. ¿Se contempla (y utiliza) el cuerpo de conocimientos disponible como la cristalización del trabajo realizado por la comunidad científica y la expresión del consenso alcanzado?

\section{Consideraciones finales...}

Es importante contextualizar la ciencia, es decir, relacionarla con la vida cotidiana, actual y futura de los estudiantes y hacer ver su interés para sus futuras vidas en los aspectos personal, profesional y social.

Es necesario que amplios sectores de la población, sin distinciones, accedan al desafío y la satisfacción de entender el universo en que vivimos y que puedan imaginar y construir, colectivamente, los mundos posibles.

Es importante acceder a los conocimientos científicos por muchas y múltiples razones, pues como dice Claxton (1994) «importan en términos de la búsqueda de mejores maneras de explorar el potencial de la naturaleza, sin dañarla y sin ahogar al planeta. 
La escuela debe por tanto afrontar el reto de proporcionar a cada persona la formación científica básica necesaria, para ser capaz de desenvolverse en su entorno enmarcándose dentro de una visión constructiva para que los estudiantes transformen y comprendan la realidad que les rodea. Teniendo en cuenta que la mayor parte de la información científica que ellos manejan procede del contexto en que se desenvuelven, se debe orientar la educación e impregnar al alumno de una cultura donde se le dé importancia a la actividad científica, a su naturaleza, sus métodos y sus relaciones con la sociedad; no podemos reducir la ciencia a los conceptos que ha producido, se debe de tener en cuenta los procedimientos y actitudes.

La escuela como agente socializador debe garantizar el acceso a la cultura científica y es la encargada de formar ciudadanos capaces de comprender, desarrollar o transformar su realidad.

La adquisición de una urgente metodología basada en el cuestionamiento científico, en el reconocimiento de las propias limitaciones, en el juicio crítico y razonado, debe insertarse en todo proyecto de desarrollo de la persona y colaborar en la formación de un ciudadano capaz de tomar sus propias decisiones, ya que prepara y favorece una actitud crítica, razonable. Como dice Gil (1996),

la influencia creciente de las ciencias y la tecnología, su contribución a la transformación de nuestras concepciones y formas de vida, obligan a considerar la introducción de una formación científica y tecnológica (indebidamente minusvalorada) como un elemento clave de la cultura general de los futuros ciudadanos y ciudadanas, que les prepare para la comprensión del mundo en que viven y para la necesaria toma de decisiones.

Es decir, se procura contribuir a formar ciudadanos responsables que viven y se desenvuelven en una sociedad cada vez más compleja en la que la ciencia y la tecnología ocupan, sin duda, un lugar fundamental en sus vidas. Como señala Izquierdo, "la auténtica educación científica debe capacitar para la crítica y debe permitir que los jóvenes consideren que su intervención en la sociedad es necesaria y va a ser posible en una perspectiva de cambio para mejorar colectivamente" (2000).

Los alumnos han de "vivir la ciencia" en la escuela, combinando las acciones propias del conocer con las que corresponden al ser, al convivir, al hacer. Por esto requiere de una epistemología y de una axiología que sean propias del contexto escolar, y que proporcionen al estudiantado un espacio de vida en el cual sea posible la utopía, la creencia íntima que, finalmente, orientará al decidir.

El centro de nuestra argumentación se fundamenta en el hecho de que para promover y estimar el desarrollo del pensamiento científico de los alumnos, es necesario tener en cuenta el sentido que cobra su implicancia en actividades de solución de problemas. Además pone de manifiesto las transformaciones esenciales que se producen en ellos como sujetos independientes y competentes; así como en los grupos, entendidos como totalidades, cuyas diferencias individuales aportan a la confluencia de objetivos comunes en un proceso centrado en la tarea y en las "condiciones de aprendizaje".

En este sentido, intentamos una aproximación a las repercusiones deseables que, para los estudiantes, deben tener los procesos de solución de problemas científicos, concebidos con fines de alcanzar transformaciones profundas, no sólo en sus estructuras de conocimientos específicos y de los recursos formales, axiomáticos y/o heurísticos; sino sobre todo, en aquellas que definen el sentido personal de esa actividad escolar y las posibilidades de operar sobre su propio desarrollo a través de ella en un ambiente de comprensión teórica de las mismas actividades y de los criterios u obstáculos para lograrlas (Carbonell \& Furio, 1987 ; Labarrere \& Quintanilla, 2006).

Para aprender la cultura de la ciencia hay que fijar el valor que la ciencia tiene, tanto para los adultos y los niños, como para nuestra cultura en la dimensión socio-histórica, en el aspecto humano y reconocer que la ciencia no goza de privilegios epistemológicos, que no es algo bueno e incuestionable. Solo si se comprende la naturaleza de la 
sociedad en la que se asienta y la naturaleza de su relación cambiante con el público de esa sociedad, se puede iniciar una revaloración de la ciencia.

Para aprender la cultura de la ciencia, hay que mejorar su comprensión pública, debe insistir no como hacer ciencia o aprender conocimiento científico, sino en indagar como leer y comprender el discurso de la ciencia de forma crítica e instruida. El aprendizaje de la ciencia como un producto cultural, como ocurre con los estudios literarios, en que los textos se abordan no solo en busca de lo que son sus contenidos o trama. Esto nos llevaría a examinarnos como se produce, se gestiona y se transforma el conocimiento científico para reconocer no solo las virtudes de la indagación científica, sino también sus defectos y limitaciones.

\section{Bibliografía}

Bateman, W. (1999). Algunos pensamientos de Piaget $y$ de Perry. Alumnos Curiosos preguntas para aprender y preguntas para enseñar. Editorial Gedisa. pp. 47-61.

Bruner, J. (1999). La educación, puerta de la cultura, Madrid: Visor.

Carbonell, F. \& Furio, C. (1987). “Opiniones de los adolescentes respecto del cambio sustancial en las reacciones químicas". En Enseñanza de las Ciencias, núm. 5, vol. 1, pp. 3-9.

Claxton, G. (1994). Educar mentes curiosas. Madrid: Visor.

Delors, J. "La educación encierra un tesoro". Informe a la UNESCO de la Comisión Internacional sobre la Educación para el Siglo XXI. Santilla ediciones UNESCO.

Durá Gilabert, P. (2006). "Analfabetismo científico”, [en línea]. Disponible en http://www.almendron.com/tribuna/?p=12668 [consultado el 23 de enero de 2007].

Fara, P. (2009). Breve historia de la ciencia. Barcelona, España: Ariel.
Fumagalli L. (2002). "La enseñanza de las ciencias naturales en el nivel primario de educación formal. Argumentos a su favor". En Didáctica de las ciencias naturales aportes y reflexiones. Ecuador. Buenos Aires: Paidós.

Giere, R. (1999). "Un nuevo marco para enseñar el razonamiento científico". En Enseñanza de las Ciencias, Núm. Extra, junio 1999, pp. 63-69.

Gil, D. (1996). Proposiciones para la Enseñanza de las Ciencias de los 11-14 años. Síntesis presentada después de la reunión técnica de Montevideo. UNESCO-OEI (documento interno).

Gil, D. (2005). ¿Cómo promover el interés por la cultura científica? Una propuesta didáctica fundamentada para la educación científica de jóvenes de 15 a 18 años. Oficina Regional de Educación para América Latina y el Caribe OREALC/UNESCO - Santiago. Década de la Educación para el Desarrollo Sostenible declarada por Naciones Unidas (2005-2014).

Harlen, W. (2002). "Evaluar la alfabetización científica en el programa OCDE para la evaluación internacional de estudiantes (PISA)", En Enseñanza de las Ciencias, núm. 20, vol. 2, pp.209-216.

Izquierdo, M. (1996). "Relación entre la historia y la filosofía de la ciencia y la enseñanza de las ciencias". En Alambique, núm. 8, pp. 7-21.

Izquierdo, M. (2000). "Fundamentos epistemológicos”. En F. J. Perales \& P. Cañal (Eds.) Didáctica de las Ciencias Experimentales, Alcoy: Editorial Marfil.

Izquierdo, M., Sanmartí, N., Espinet, M. y García, P. (1997). Caracterization and Foundation of School Science.

Labarrere, A. \& Quintanilla, M. (2006). "La evaluación de los profesores de ciencias desde la profesionalidad emergente". En Enseñar ciencias en el nuevo milenio. Retos y propuestas. Quintanilla, M. \& Adúriz-Bravo (Eds). Santiago de Chile: Ediciones PUC. 
Malaguzzi, L. (2001). La educación infantil en Reggio Emilia. Barcelona: Octaedro-Rosa Sensat.

Mercé, P. "El analfabetismo científico, un mal curable", [en línea]. Disponible en http:// digital.el-esceptico.org/leer.php?id $=2100$ \&autor $=635 \&$ tema $=145$ [consultado el $23 \mathrm{de}$ enero de 2007].

Osborne, J. C., et al. (2003). "What 'ideas-aboutscience' should be taught in school science? A Delphi study of the expert community", En Journal of Research in Science Teaching, núm. 40, vol. 7, pp. 692-720.

Pérez Gómez, A. (1996). Las funciones sociales de la escuela: de la reproducción a la reconstrucción crítica del conocimiento y la experiencia. Comprender y transformar la enseñanza. Ediciones Morata.
Quintanilla, M. (2006). "Identificación, caracterización y evaluación de competencias científicas desde una imagen naturalizada de la ciencia”. En: Enseñar Ciencias en el nuevo milenio. Retos y propuestas. Quintanilla,M. \& Adúriz-Bravo (eds). Santiago de Chile: Ediciones PUC.

Sanmartí, N. (Coord.) (2003). Aprendre ciències tot aprenent a escriure ciència. Barcelona: Ediciones 62.

Stenhouse, W. (1985). Investigación y desarrollo del currículo. Madrid: Morata

Zabalza, M. A. (1996). Calidad en la educación infantil. Madrid: Narcea. 